\title{
Reliable operant apparatus for fish: Audio stimulus generator, response button, and pellet-dispensing nipple
}

\author{
AVA R. CHASE and WINFIELD HILL \\ Rowland Institute for Science, Cambridge, Massachusetts
}

\begin{abstract}
As part of ongoing research into the ability of koi to categorize complex auditory stimuli, we have had to develop novel apparatus. The stimulus generator presents sound from two CD drives under computer control through a new underwater speaker. The operant manipulandum is a horizontal button that eliminates spurious triggering by water turbulence and problematic response topographies. The button's design has also been adapted for use by tilapia in an aquacultural food-preference study. The feeder uses a nipple to dispense food pellets reliably under water. In this paper, the apparatus is described in detail. Also discussed are methodological issues related to its design, as well as its usage in a pilot study in which koi learned to discriminate music from silence by using a single manipulandum with food reinforcement.
\end{abstract}

We have been studying the ability of fish-specifically koi (Cyprinus carpio) - to discriminate and categorize complex auditory stimuli such as music. In this paper, we describe both the apparatus that we have developed and a preliminary experiment that demonstrates the use of the apparatus and illustrates some methodological issues. In designing the apparatus, we were guided by ethological as well as practical considerations.

In designing our aquarium, we were influenced by the fact that koi are social animals. Living in isolation can be stressful in itself, but transportation between a home tank and a separate experimental tank can be stressful and experimentally impractical, especially once fish have grown to a length of more than a foot and a weight of several pounds. Therefore, our aquarium consists of a group home tank that can be partitioned into experimental and holding areas.

We also developed a response button and feeder because we needed reliable equipment. It has commonly been reported that response systems can be triggered by a fish's body accidentally colliding with the manipulandum (Ardon, Grant, \& Cowey, 1973), or by water turbulence created by fin or body movements. In early experiments, we tried flexible paddles made from Kynar piezo film sen-

We are grateful to Holly Perry, whose artful and technical hand made the illustrations illustrious. We thank Matt Maltzman for his support and his comments on the manuscript. We also thank Don Rogers for making the tank, Peter Kenny for machining the instruments, Angel Peterchev for instrument refinements, and Bob Newton for making the electronics. Thanks are due Chris Stokes for helping to prepare the manuscript. We thank the Rowland Institute for Science for supporting this work. We are grateful to Allan Neuringer for his encouragement and for his comments on the manuscript, and to the anonymous reviewer for helpful feedback. Correspondence concerning this article should be addressed to A. R. Chase, Rowland Institute for Science, 100 Edwin Land Blvd., Cambridge, MA 02142 (e-mail: chase@)rowland.org). sors (Yan \& Popper, 1991) but found them to be excessively sensitive to water turbulence. Next we tried rigid plastic paddles mounted at the ends of $30-\mathrm{cm}$ rods that were instrumented with strain gauge sensors. Even when each rod and paddle were enclosed in a protective housing with only a portion of the front surface of the paddle exposed, we continued to have trouble with water turbulence and accidental actuations from miscellaneous body parts. Stiffening the motion with dense foam blocks eliminated the problem only partially. This susceptibility of apparatus to false triggering creates a possibility of functionally noncontingent reinforcement. In a previous two-choice visual discrimination experiment with one of the present subjects, the fish stopped mouthing the keys altogether, forcing us to redefine responding in terms of spatially distinctive swimming patterns. In another experiment, we observed response topographies that were incommensurable between subjects and sometimes difficult to interpret in individual subjects. For example, one fish would operate the paddle by pecking it, but another would begin its characteristic response at the far end of the tank, swim near the surface toward the paddle and swoop down past it, brushing the paddle with its tail as it left.

Especially in an automated experiment, the apparatus itself must help prevent unacceptable response topographies, so that it registers only what a human observer would agree is a response. We have found that suitable geometry and electronics can reliably define a response to be contact with a fish's mouth as opposed to any other part of its body. Our latest manipulandum is a horizontal button that virtually eliminates spurious triggering by water turbulence and topographically unconstrained responding.

Food delivery poses special problems in aquatic environments as well. Solid food frequently gets stuck when delivery channels become wet, causing the animal to fail 


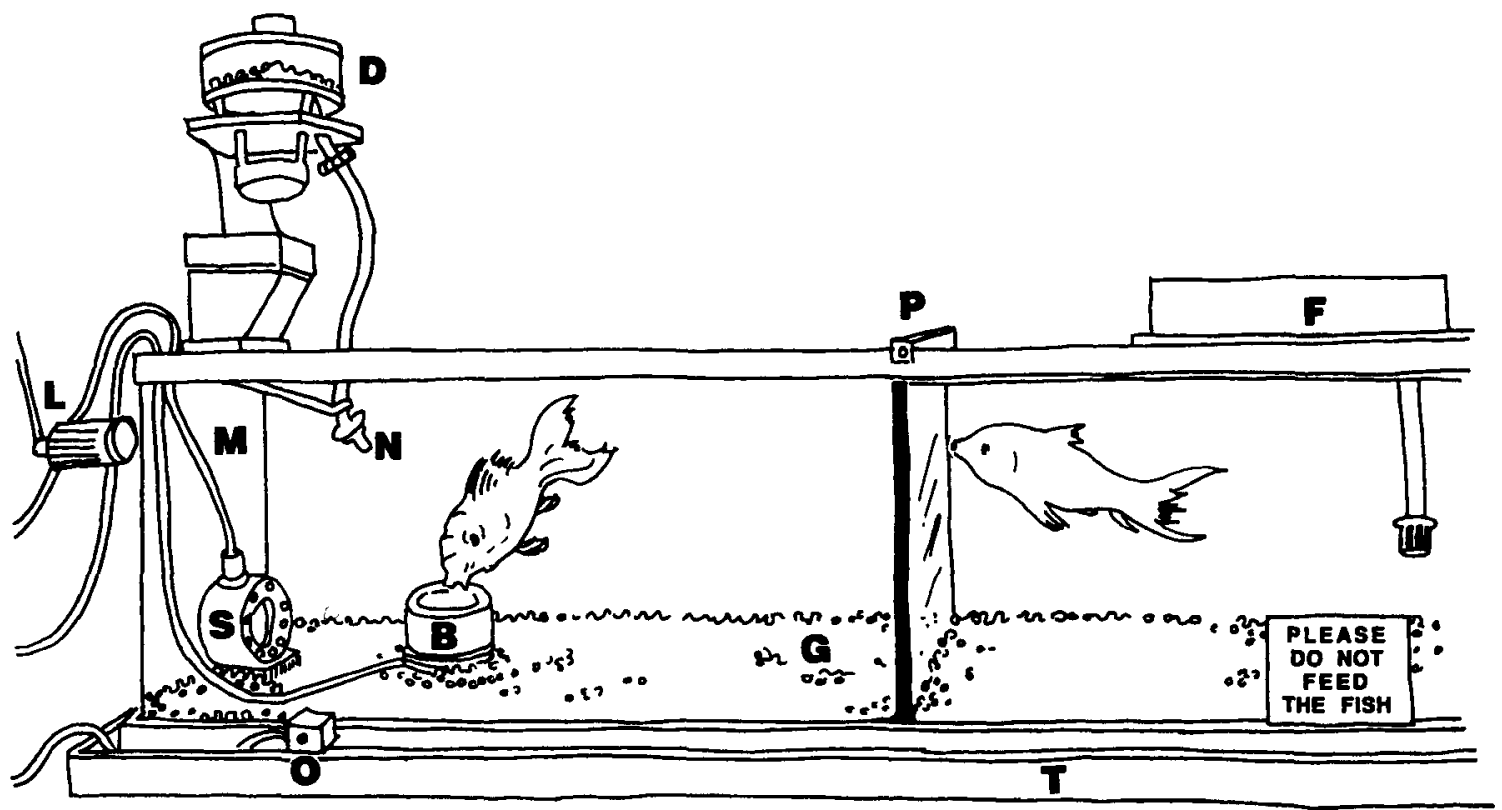

Figure 1. The working end of the experimental tank, as seen by the video camera (not shown), includes partition $P$ (at the silicone joints), filter $F$, tank tray $T$, gravel bed $G$, speaker $S$, response button $B$, light $L$ (the conditioned reinforcer), which shines through light-diffusing mylar $M$ onto nipple $N$, pellet dispenser $D$, and LED $O$, which shows the camera that the computer has registered a response.

to receive a reinforcement. Inadvertent unreliability in the dispensing of food is sometimes finessed retrospectively in the labeling of the reinforcement schedule as intermittent (Goldman \& Shapiro, 1979). It appears that most food delivery apparatus for fish, however, simply drops the food into the tank (Beach, Baker, \& Roberts, 1986; Cuenca \& De La Higuera, 1994). Even when the food makes it into the water, a fish can lose track of it and become distracted from the experimental task while foraging for the lost morsel. With koi, floating food pellets, even if confined to a designated area, can disrupt an experiment, because a fish can have difficulty homing in on the pellet and can then spend an inordinate amount of time bobbing for it. Our feeder presents food pellets to the fish under water through a slightly modified nipple, where they remain until the fish sucks them out.

In auditory experiments with terrestrial nonhuman subjects, a design feature found to significantly facilitate auditory-discrimination learning has been the adjacency between the response manipulandum and the sound source (D'Amato \& Salomon, 1982; Harrison, 1992; Segal \& Harrison, 1978). Our underwater speaker was designed to be conspicuously visible to the fish, and the response button (which was conceived after the speaker was built), was designed so that it could be contiguous to the sound source.

\section{APPARATUS}

\section{The Tank}

Our 3 subjects live in a 450 -L ( 120 -gal.), $210 \times 52 \times$ $41 \mathrm{~cm}$ tank, which is large enough to be partitioned dur- ing experimental sessions into a working area for 1 fish and a holding area for the others. The partition can be positioned anywhere along the length of the tank, but we have consistently worked with an experimental chamber $80 \mathrm{~cm}$ long. The partition can be opaque or transparent; the one in current use is clear plastic, and the social interactions across it have been an interesting - and possibly significant -feature of our experiments. Koi seem to experience less stress and to perform better if they have visual access to other shoal members.

The tank was designed to allow acoustic as well as visual isolation of the test chamber from the holding area. The walls and floor are not single pieces of glass; each rather comprises two pieces, separated by a vibrationattenuating silicone joint $80 \mathrm{~cm}$ from one end. In principle, a sound-dampening partition can be positioned at this joint to minimize acoustic transfer between the two chambers, but we have yet to implement this feature. Our tank does not provide any special isolation from external stimuli, which have thus far not measurably disturbed our subjects. The glass elements are held in a metal framework. The tank rests on a standard laboratory bench, which needed structural reinforcement to support the weight. The physical layout of the tank is shown in Figure 1.

\section{The Stimulus Presentation System}

The audio stimulus presentation system takes sound from two standard SCSI CD drives under computer control. Two 620- $\Omega$ resistors are used in series with the stereo outputs of each CD drive to convert stereo sound to mono and to mix the signals when both $\mathrm{CD}$ drives are 


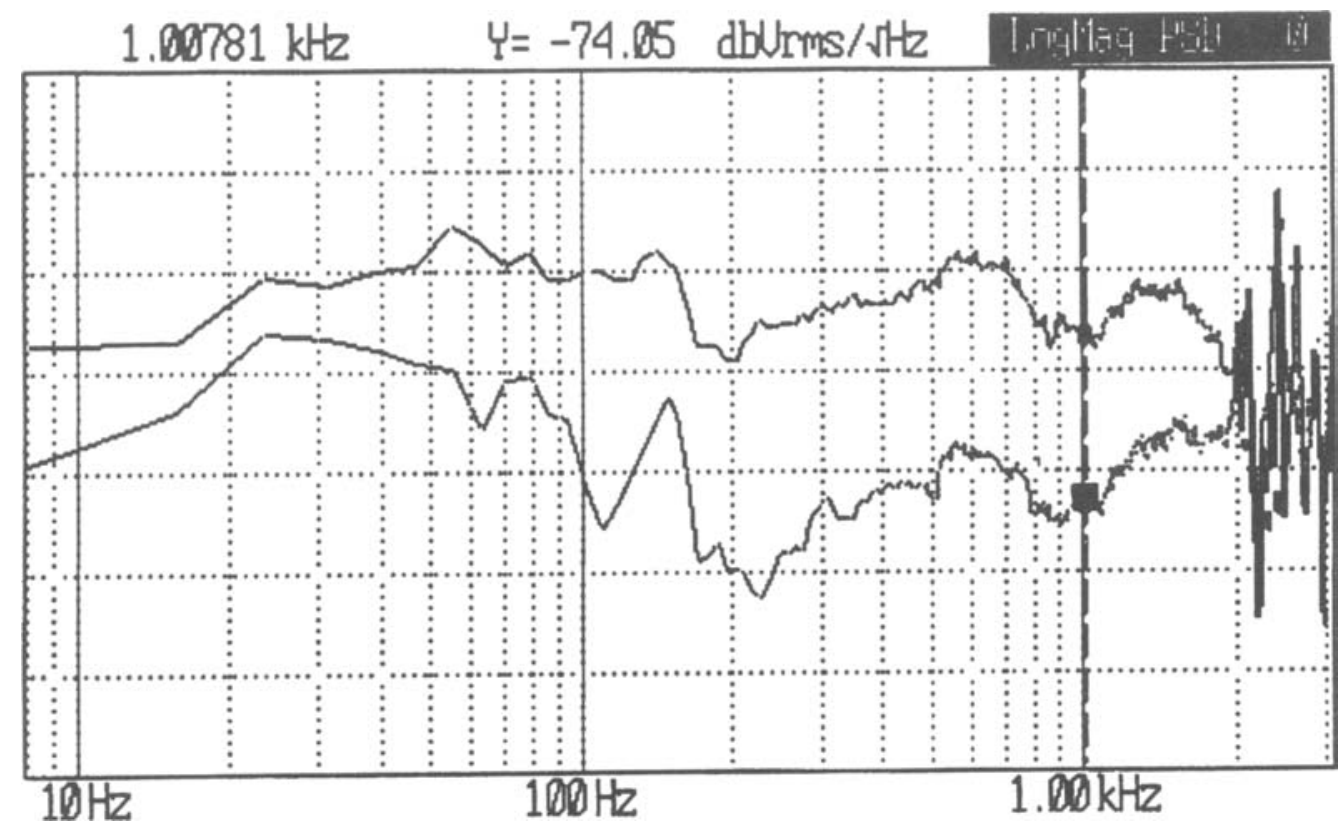

Figure 2. Frequency response characteristics of the tank from $7.81 \mathrm{~Hz}$ to $3.125 \mathrm{kHz}(10 \mathrm{~dB}$ per division), measured with the divider in place, using a white-noise source and a laboratory-standard hydrophone. Measurements were made $15 \mathrm{~cm}$ from the speaker (top trace) and in the middle of the experimental chamber (bottom trace). The cursor dot is at $-74 \mathrm{~dB}$ (re: $1 \mu \mathrm{V}$ ) noise-voltage density, or $137.5 \mathrm{~dB}$ (re: $1 \mu \mathrm{Pa}$ ), given the TC4013 hydrophone's $-211.5 \mathrm{~dB}$ (re: $1 \mathrm{~V} / \mu \mathrm{Pa}$ ) calibration data.

used. An eighth-order high-pass filter was included to provide the option of limiting possible lateral-line stimulation in fish by music frequencies lower than $200 \mathrm{~Hz}$, where this sensory system is most responsive (Coombs, Janssen, \& Montgomery, 1992). Volume-compression circuitry (DBX Professional Products, Model dbx 163A) helped control for the possibility of subjects' discriminating between the stimuli from each $C D$ drive merely on the basis of differences in dynamic range. A University Sound (Sylmar, CA) UW-30 underwater speaker has been used by other experimenters (Fay, 1992; Yan \& Popper, 1991), but we found it to be too large $(1,735 \mathrm{cc})$ and to have a poor frequency response. (The manufacturer recommends a baffle with a 12-cm-diameter hole and a large wetniche behind the speaker, which was not practical for our fish tank. Moreover, we wanted visual adjacency between the sound source and the response manipulandum, which was not possible with such a scheme.) We used a 10-cm 10-W speaker (Radio Shack 40-1197), which we mounted in a 14-cm-diameter plastic underwater housing (Lexan, 1.25-cm-thick wall, 500cc inside air space) with a stainless steel weight screwed to the base. The speaker is driven by a 35-W P.A. amplifier (Radio Shack MPA-45), and the sound is coupled to the water with a thin, flexible membrane (Vistafoil; Demco, Fresno, CA). The enclosure is machined from a single piece of plastic, to ensure sealing integrity. Two facing grooves allow the membrane to be sandwiched between two $O$ rings, secured with a clamp ring and eight SS bolts on a $12.7-\mathrm{cm}$ bolt circle. This arrangement has remained watertight for several years, without any maintenance.

The frequency responses of the amplifier, speaker, and tank system were measured with a white noise source and spectrum analyzer (SRS Model SR780; Sunnyvale, CA) and a laboratory-reference calibrated hydrophone (Reson Model TC 4013; Goleta, CA) and were found to be within $10 \mathrm{~dB}$ from $150 \mathrm{~Hz}$ to $3 \mathrm{kHz}$ near the speaker (Figure 2). Quiet nodes existed near the center of the tank, outside the experimental area (in the holding area). Hydrophone observation also revealed areas of heightened sound levels in the tank, such as the corners. The sound volume was set +60 to $+100 \mathrm{~dB}$ (re: $1 \mu \mathrm{Pa}$ ) in the experimental area. The maximum is about $50-60 \mathrm{~dB}$ above the hearing threshold for carp (Fay, 1988; Popper, 1972), and well below the 149-dB level where Popper and Clarke (1976) demonstrated that hours of exposure produced up to a 30-dB temporary decrease in auditory sensitivity in goldfish.

During the hydrophone measurements, it was found that a very loud buzz (up to $150 \mathrm{~dB}$ [re: $1 \mu \mathrm{Pa}$ ] near the air hose) was injected into the tank by the air pump. Consequently the pump was turned off well before each experimental session. Quiet gurgles from the purification filters were acceptable.

\section{The Response Button}

The operant manipulandum, dubbed the Chase fish button, overcame the problems encountered with previous designs. The cylindrical assembly lies on the bottom 


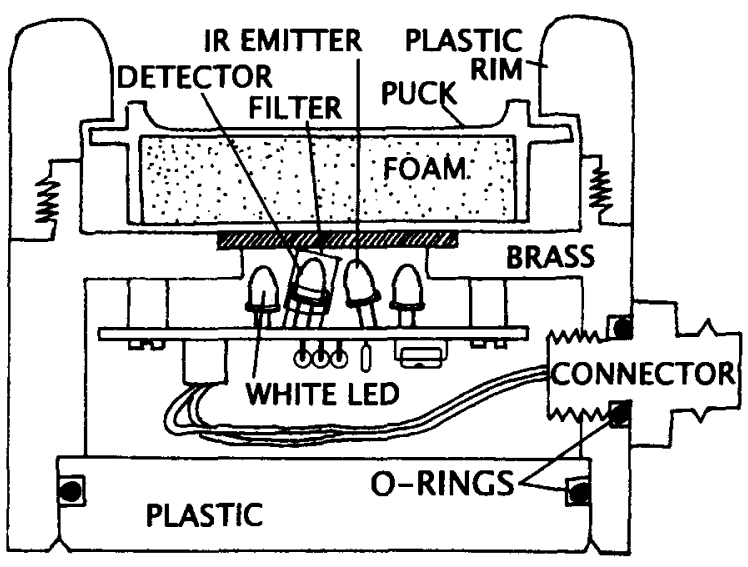

Figure 3. The Chase fish button features a response puck made buoyant by plastic foam and held in place by a rim.

of the tank in the gravel bed and it has just one moving part (see Figure 3) - a circular "puck" made of translucent plastic, which floats by virtue of a closed-cell polyethylene foam insert. The puck is designed to be depressed downward and to be restored by flotation alone and is constrained and protected by the rounded top rim of the housing.

Two sizes of the Chase fish button have been constructed. The larger, intended for use with fish $20 \mathrm{~cm}$ or more in length, such as the koi subjects in this experiment, is a 10 -cm-high $\times 12$-cm-diameter machined assembly, which lies stationary, pushed about $1 \mathrm{~cm}$ into the gravel on the bottom of the tank. The top half of the assembly is a threaded (black) plastic rim with an $8.25-\mathrm{cm}$ inside-diameter (ID) hole, through which the fish can reach the (clear sandblasted) 3-cm-thick puck. The rim's threads allow easy removal for quick inspection and cleaning. The puck is hollowed out underneath to receive the buoyant insert. A $9.55-\mathrm{cm}$ ID undercut in the rim keeps the puck at least $2 \mathrm{~cm}$ below the top of the rim, so that in order to respond, a fish must swim down into the housing and push down on the puck. This geometry discourages operation by anything other than the fish's mouth.

The puck has a $9.5-\mathrm{cm}$ outside-diameter (OD) flange to keep it centered in the undercut rim with about $0.03 \mathrm{~cm}$ of clearance all around, and an 8.06-cm OD outer ridge to help prevent any jam by directing gravel dropped by the fish away from the clearance edge. To allow the displaced water to move freely when the puck is pushed, the flange on the puck has 12 scalloped cutouts. We have also machined pucks with a raised "target" in the center, but to date this has been tried only on the smaller version, used with tilapia.

The puck and its flotation foam are translucent and can be illuminated by eight white LEDs (Nichia America; Mountville, PA) housed under a glass window below the puck. Whenever a sufficient displacement of the puck is detected, these lights come on for about 2 sec. This provides instant feedback to the subject that a response has been registered, as distinct from signaling any particular reinforcing or other consequence of the response. This usage is analogous to the tactile feedback provided by some keyboards.

Displacement of the puck is sensed through measurement of optical backscatter with an infrared (IR) emitter LED and a phototransistor detector (Radio Shack 27-143 and 27-145 A), located under the glass window in the electronics chamber about $0.75 \mathrm{~cm}$ below the puck. The LED is positioned to illuminate the underside of the puck's flotation foam, and the adjacent detector senses the backscattered light. The detector is surrounded by black heat-shrink tubing and is fitted with an IR filter (made from unexposed, developed Kodak Ektachrome slide film) to eliminate the white light emitted from the response LEDs. The IR emitter is driven with a $5-\mathrm{mA}$ $1.8 \mathrm{-kHz}$ square wave from a cmos 555 square-wave oscillator (see Figure 4) and the light-sensor signal is amplified with a $2.2 \mathrm{k}$ transresistance amplifier. A phasesensitive-rectifier lock-in detector (Horowitz \& Hill, 1990 ) is constructed with a cmos switch (selecting either the signal or its inverted form, synchronously with the LED drive) and a low-pass filter with a 10-msec time constant. With a postdetector amplifier $(\mathrm{G}=21)$, this yields a lock-in signal roughly proportional to the position of the puck, at a range of up to $1 \mathrm{~cm}$. Thereafter, a high-pass filter $(2.5 \mathrm{sec})$ removes the steady position component, leaving a change-in-position signal, and a second-order low-pass filter $(25 \mathrm{msec})$ helps eliminate overhead lighting interference. A comparator with a 100msec pulse-length stretcher prepares the final signal for the computer. Six wires with an underwater cable and connectors (Impulse Enterprise, San Diego) connect the underwater electronics, with O-ring seals used for integrity.

The displacement sensitivity (typically $0.2-1 \mathrm{~mm}$ ) required in order to activate the button can be adjusted by changing the comparator threshold with a knob. The buoyancy force can be adjusted by changing the thickness of foam in the puck. The larger koi version can be depressed by about $75 \mathrm{~g}$ of force; a small tilapia version (9-cm-high $\times 9$-cm-diameter assembly with a 6 -cm rim opening) requires about $15 \mathrm{~g}$.

In our years of experience with multiple versions of this button, we have not observed spurious triggering due to water turbulence, inappropriate body contact, gravel dropped on the puck, or any other cause.

\section{The Feeder}

Our feeder system is the only one that we know of in which a nipple is used to dispense solids. We use a standard pellet dispenser (Gerbrands G5120, or MED Model ENV 203-20; Georgia, VT) that comes fitted with an outlet to which we clamp a 5/8-in. (16-mm) plastic tube. Sinking pellets (Noyes, Formula J/G, $20 \mathrm{mg}$ ) fall through 


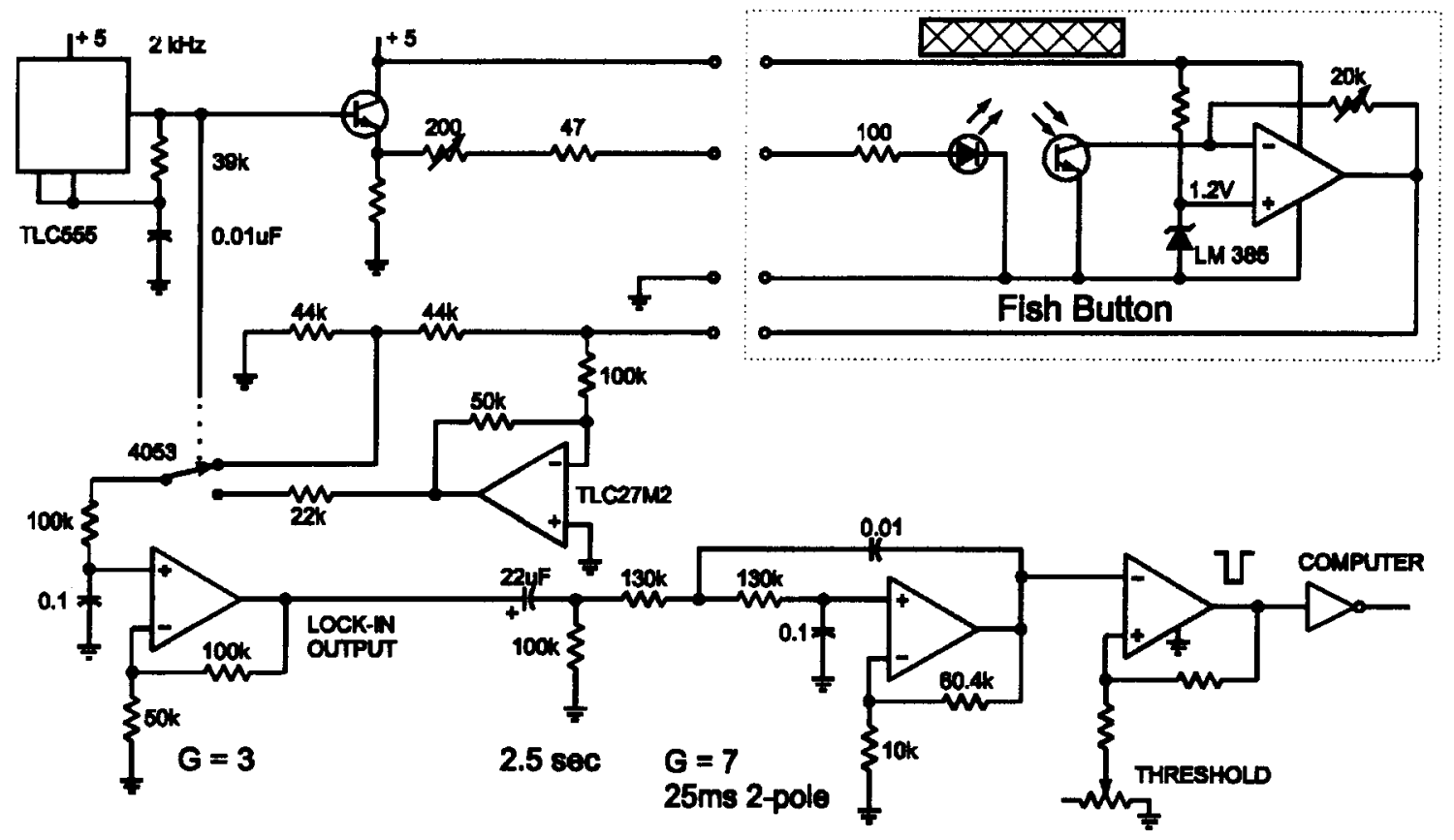

Figure 4. Circuit diagram of the electronics in the Chase fish button and its out-of-tank control box. The power and timing circuitry that drives the white LEDs is not shown.

the tube, which terminates in a baby-bottle nipple (HealthFlow, Stage 1; Johnson \& Johnson), that is positioned with cable ties from 2 to $10 \mathrm{~cm}$ below the surface of the water. In the end of the nipple we have made X-cut slits, along with a slight enlargement of the hole. The hole is kept small enough to prevent a pellet from falling out, while the slits ensure that it can be sucked out.

The tube must be securely fastened both to the dispenser and to the nipple to prevent a fish from pulling the apparatus apart; machine-tensioned cable ties work well. Because of wear and tear, we replace the nipple periodically.

A small halogen light (Sunex Series 700; Natick, MA) external to the tank and diffused with frosted glass functions as a conditioned reinforcer (CR) indicating the presence of food and is arranged to backlight the nipple and pellet to increase their visibility.

\section{The Control System}

Experiments with this apparatus are controlled by a program written in compiled BASIC (Microsoft PDS, Version 7.1) and running under MS-DOS (Version 6.2) on an Intel $386 \mathrm{SX}$ computer. The program's CD-drive access was coded with a Developer Kit library (Creative Labs, 1994). The computer sequences the stimulus presentations, continuously monitors the response button, times all signals with $55-\mathrm{msec}$ resolution, imposes the programmed conditional consequences of responding and nonresponding, operates the pellet dispenser and CR light (via an ac relay), and displays all activity in real time and writes it to disk. The details of the protocol for a given experiment are implemented in the code and are customized by editing the values of constants and variables. Operational refinements can be included in the programming (e.g., to prevent unintended quiet periods, before the beginning of a music-playing interval, the computer advances the CD drive to the next track if insufficient time remains in the current track).

A Hi-8 camera videotapes the experimental chamber and the part of the home chamber nearest the partition (see Figure 1), records the audio signal being fed to the underwater speaker, and feeds a monitor in the experimenter's office.

\section{APPLICATION AND FEASIBILITY STUDY: DISCRIMINATION OF SOUND VERSUS SILENCE}

One of our research goals has been to determine whether fish can learn to discriminate music on the basis of style or genre, as people and even pigeons can (Porter $\&$ Neuringer, 1984). The study presented here was a methodological proof of concept, designed to test the effectiveness of a single-manipulandum protocol and to verify the reliability of our new apparatus. We used stimuli whose categorical discriminability was not in question-namely, music and silence. The procedure was simply to present randomly timed intervals of music $(\mathrm{S}+)$ and silence $(\mathrm{S}-)$, in alternation, and to reinforce, with food, each response that was made in the presence of music.

Consider how data from the single manipulandum might demonstrate a discrimination. Given that the con- 
ditions are continuous reinforcement signaled by music and extinction signaled by silence, a mere difference in response rate would not suffice, because even a deaf subject could exhibit a rate differential simply by discriminating the prevailing reinforcement contingency. Thus, we let our primary criterion be that a statistically significant majority of $S$ - intervals show no responding whatsoever while a significant minority of positive stimulus $\mathrm{S}+$ intervals show no responding. The response rate during $\mathrm{S}+$ intervals could primarily be a function of reinforcement scheduling, so it is less important that responding be especially high during $\mathrm{S}+$ intervals than that it be minimal across $\mathrm{S}$ - intervals.

Clearly a crucial issue is whether in the absence of S+ our subjects would have any problems inhibiting responding on the new button. The possibility that responding might intrinsically have minor aversive properties, which might bias the response rate downward, is tolerable, but responding must not be intrinsically reinforcing. Also, because our fish are bottom feeders and the button lies horizontally on the gravel bed, the operandum must not evoke "ethological" mouthing that could be mistaken for a discriminative response (A. A. Wright, 1992).

\section{Method}

Subjects. The subjects were three koi (Cyprinus carpio), purchased from a local pet store, who at the time of the experiment ranged in age from 7 to 11 years and had lived together in our lab for 5 years. Pepi was $36 \mathrm{~cm}$ from snout to base of tail and weighed approximately $1.8 \mathrm{~kg}$; Beauty and Oro were about $7 \mathrm{~cm}$ shorter. All 3 had previously been exposed to many kinds of music in a study (unpublished) of sensory reinforcement. Beauty had also served in a visual categorization experiment with a twomanipulandum discrimination procedure.

Apparatus. The apparatus was as described previously, but without the high-pass filter, which was omitted because we were not concerned about possible lateral line involvement.

Training procedure. Training began with group sessions. A nipple affixed to a piece of tubing was repeatedly loaded with a pellet and offered to individual fish until all had learned to suck out the pellet; during this phase, we perfected the modifications to the nipple. Next we connected the nipple's supply tube to the automatic pellet dispenser, which we triggered manually, and within minutes all 3 fish had learned to eat from this stationary feeder. We then introduced an external light as a $\mathrm{CR}$; it was aimed to backlight the nipple and pellet. At first we kept this light on manually from the time at which a pellet was dispensed until it was eaten, but we soon automated it to come on for $3 \mathrm{sec}$ after a pellet had been dispensed.

Next we installed the button at one end of the tank and suspended the nipple about $5 \mathrm{~cm}$ above it on the side nearest the wall. With all 3 fish confined to the experi- mental chamber by the partition, we shaped the buttonpressing response, using the technique of "learning by following" (Bullock \& Neuringer, 1977; Neuringer \& Neuringer, 1974). The experimenter lured Pepi (a highly interactive fish) down to the button with her hand and with her index finger depressed the puck, which triggered the CR. Only two demonstrations were needed, after which Pepi responded more than 60 times in that first 2-h session. (A related technique has quickly taught buttonpressing to tilapia that were less familiar with human hands than our koi were. With the button's displacement sensitivity temporarily increased, pellets are dispensed directly onto the puck through a clear tube. The fish follows the pellet down and eats it off the puck, adding enough pressure to trigger the CR.) To operate our button, a fish must adopt a nose-down position, which among carp communicates the location of food (Magurran, 1984). Pepi's behavior appeared to facilitate the acquisition of buttonpressing by Oro and Beauty (Zentall \& Levine, 1972). Oro in particular would swim in close formation with Pepi as the latter pressed the button and moved up to feed at the nipple. Fish have been shown to learn operant responses better in groups (Welty, 1934; D. E. Wright \& Eastcott, 1981).

Oro had trouble operating the button until we stuck a fan-folded piece of red tape on the puck as a target. After a few days the tape was removed, and Oro's newfound proficiency persisted. However, he developed the habit of picking up gravel and dumping it on the puck, where he would repeatedly suck it up and spit it out in a kind of foraging behavior. Such mouthing of gravel on a horizontal button by a natural bottom forager raised concerns about "instinctual drift," in which "learned behavior drifts towards instinctual behavior" (Breland \& Breland, 1961), but we detected no such effect on operant buttonpressing.

Over the course of several sessions, we moved the nipple higher until only its tip remained under water. This minimized both the pellet's delivery time and its chances of sticking to moisture in the tube. Moving the nipple was not disruptive or disorienting to the fish, because with the firm establishment of the CR the fish immediately moved to the part of the water column that was most intensely illuminated. However, increasing the distance between the button and the nipple forced an end to group training, because Oro learned to hover near the nipple and steal pellets earned by Pepi and Beauty.

Before we abandoned group sessions, we introduced the speaker and continuously played "mellow" music (e.g., The Sounds of the Grand Canyon; Orange Tree Productions, Agoura Hills, CA).

When we began individual sessions, responding was disrupted until we replaced the opaque partition with a transparent one. This is not the first study in which it appeared necessary for an experimental fish to have visual contact with others (Yan \& Popper, 1992).

Individual training sessions were terminated at $2 \mathrm{~h}$ or 80 reinforcements, whichever came first. Pepi consis- 


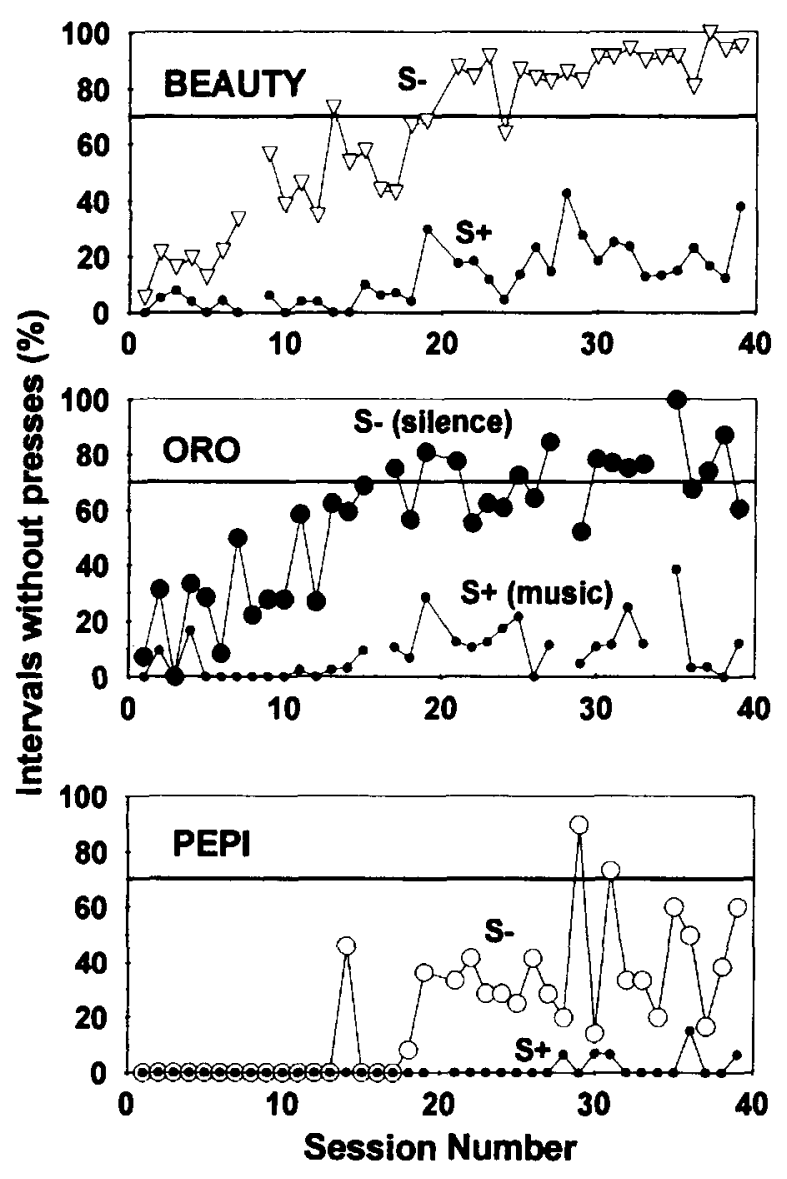

Figure 5. Percentage of $S+$ and $S-$ intervals during which the fish did not press the button at all. A comparatively high percentage of nonresponding for $S-$ indicates success; the $70 \%$ criterion line is shown.

tently reached the 80 -pellet limit in under $35 \mathrm{~min}$. Beauty took longer to extract pellets from the nipple and averaged 70 reinforcements. Oro's gravel-dumping did not activate the button, but it took time, and as with Beauty, time rather than reinforcement count terminated his sessions. After 12 individual sessions, all 3 fish were sufficiently trained on the apparatus to begin the discrimination experiment.

Experiment. Music and silence alternated in randomlength intervals (typically between 40 and $90 \mathrm{sec}$ ), and each press of the button in the presence of the $\mathrm{S}+$ (music) was reinforced. Two refinements were added. First, because the pecking response involves moving the fish's entire body toward the button and thus can be slow, a $1-\mathrm{sec}$ grace period was added after $\mathrm{S}+$ offset to permit the reinforcement of responses that were initiated in the closing moments of the $\mathrm{S}+$. Second, to ensure that the onset of the $\mathrm{S}+$ would never function as either a conditioned or a sensory reinforcer for responding during the $\mathrm{S}-$, any response that occurred within $20 \mathrm{sec}$ of the scheduled end of the $S-$ prolonged the $S-$ for $20 \mathrm{sec}$ from the time of the response. Although normally a much shorter postponement might suffice, we chose $20 \mathrm{sec}$ because of our subjects' prior experience of keypressing during silence in an experiment in which music appeared to function as a sensory reinforcer. The typical session ended with the music interval during which the 50 th reinforcement was given. A subject might earn a few more than 50 pellets in the session, because priority was given to not truncating the final interval of music. Unlike in the training procedure, there was no fixed time limit for sessions.

The S + was a John Lee Hooker CD, Blues Before Sunrise. The $\mathrm{CD}$ would cycle through, continuing from where it had previously left off, except that the remainder of a track would be skipped if it was shorter than the programmed duration of the upcoming $S+$ interval.

Since the purpose of this procedure was more to establish the suppression of responding during the $S-$ than a high rate of responding during the $\mathrm{S}+$, the goal was zero responses in at least $70 \%$ of the silent intervals in a single session. This criterion also ruled out cuing from the availability of the reinforcer.
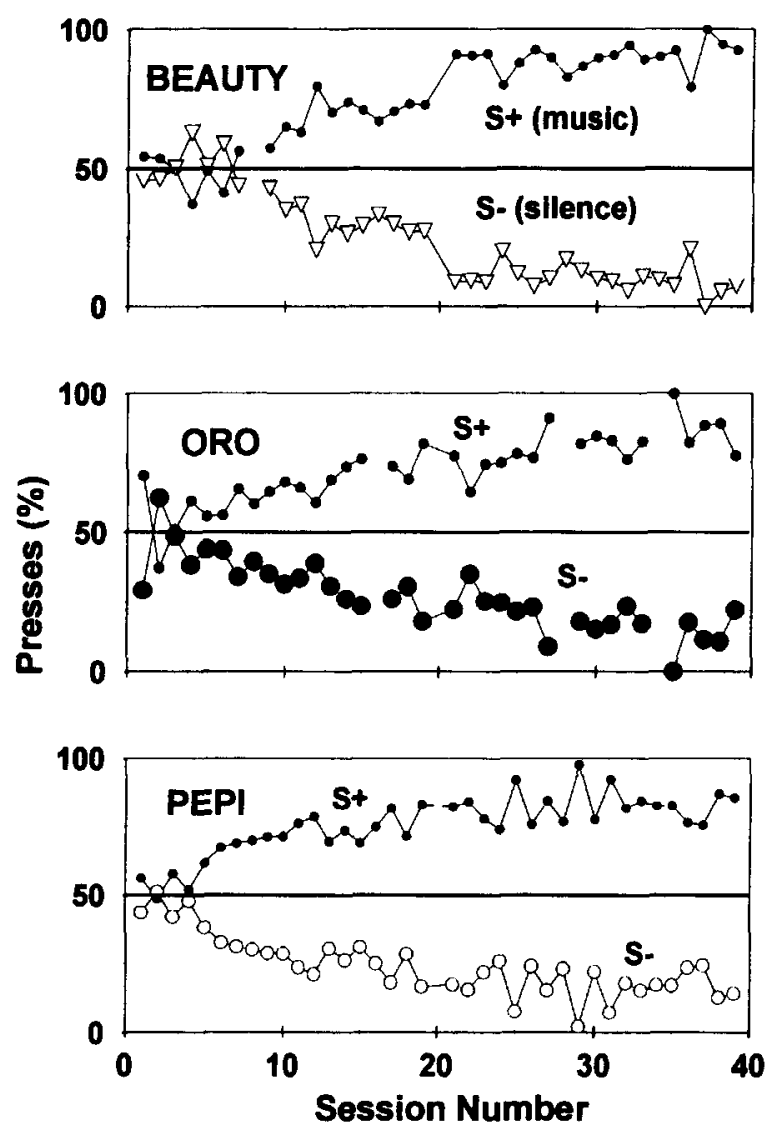

Figure 6. Buttonpressing activity for 38 sessions (Beauty missed 1 session, and Oro missed 3): the percentage of all the presses made while music is playing $(\mathrm{S}+)$ versus presses during silence $(S-)$. 


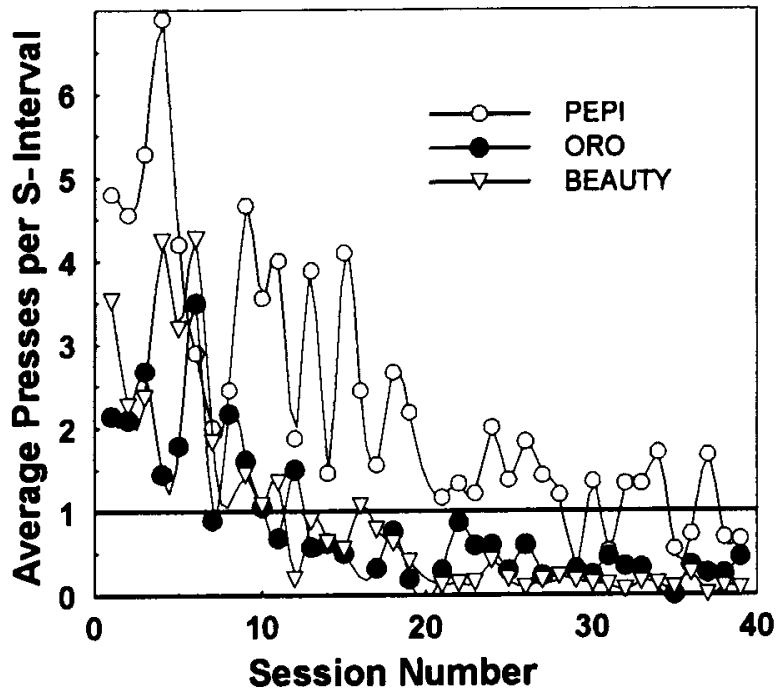

Figure 7. A session average of less than one press per $S-$ interval means that many of these intervals had no presses.

\section{Results and Discussion}

The experiment ran for 38 sessions, by which time 2 of the 3 fish, Beauty and Oro, met the " $70 \%$ no-response in S-" criterion (see Figure 5). Pepi did not meet the criterion. However, this fish almost never failed to respond in $\mathrm{S}+$ intervals ( $1.1 \%$, average over all sessions), in contrast to $\mathrm{S}-$ intervals, where the percentage of intervals with no responses was appreciably higher $(21.8 \%)$. Pepi seemed able to discriminate between the $\mathrm{S}+$ and $\mathrm{S}-$ but less able to inhibit responding.

Unlike Beauty and Oro, who averaged $70 \mathrm{~min}$ per 50 pellet session, Pepi finished in just 23 min. During $\mathbf{S}+$ intervals, Pepi's responding was fast and repetitive, a pattern that carried on beyond the cessation of the S+. Observational analysis and latency data (on responses occurring within $3 \mathrm{sec}$ after the offset of music) suggest that "momentum" was a contributing factor in Pepi's inability to meet the suppression criterion.

Figure 6 shows all the presses by all 3 fish, without regard to stimulus intervals. Over the course of three to seven sessions, random behavior evolved into clearly differentiated responding.

Figure 7 shows the responses of all subjects during the $\mathrm{S}-$. In the beginning all fish showed a high response rate, but Beauty and Oro soon clearly discriminated, and even Pepi's responding during the $\mathrm{S}-$ exhibited a downward trend.

Figure 6 demonstrates discrimination in all subjects, but it makes it difficult to see differences between subjects. Figures 5 and 7 reveal significant individual differences, especially between Pepi and the other fish, and show that all subjects discriminated on the basis of sound and not the prevailing reinforcement contingency.

In trying to ascertain an animal's cognitive capabilities, experimental requirements frequently tax a subject's abilities to the point at which positive results cannot be attained (A. A. Wright, 1992). It has also been reported that food reward procedures for studying hearing abilities in fish have been ineffective because of waning motivation over lengthy sessions and food delivery problems (Stebbins, 1983). By contrast, our results indicate that our experimental design is well suited to koi, whose motivation appears to be maintained at a high level; in fact, it is difficult to get a subject out of the experimental chamber after a session, and it is difficult to keep the other two fish out when one is trying to isolate another subject. As for reliable food delivery, the value of the nipple cannot be overemphasized. The apparatus and protocols described in this paper appear to be well-suited for discrimination experiments in which fish must respond to auditory stimuli whose categorical discriminability is in question.

\section{REFERENCES}

Ardon, J. W., Grant, P. T., \& Cowey, C. B. (1973). A system for the quantitative study of the learning capacity of rainbow trout and its application to the study of food preference and behavior. Journal of Fish Biology, 5, 625-636.

BeACH, M. A., BAKER, G. E., \& RoberTs, M. G. (1986). An accurate demand feeder for fish, suitable for microcomputer control. Physiology \& Behavior, 36, 397-399.

BRELAND, K., \& Breland, M. (1961). The misbehavior of organisms. American Psychologist, 16, 681-684.

Bullock, D., \& NeURINGer, A. (1977). Social learning by following: An analysis. Journal of the Experimental Analysis of Behavior, 25, 127-135.

Coombs, S., Janssen, J., \& Montgomery, J. C. (1992). Functional and evolutionary implications of peripheral diversity in lateral line systems. In D. B. Webster, R. R. Fay, \& A. N. Popper (Eds.), The evolutionary biology of hearing (pp. 267-294). New York: Springer-Verlag.

CREATIVE LABS (1994). Developer kit for sound blaster series (2nd ed.). Milpitas, CA: Author.

Cuenca, E. M., \& DE la Higuera, M. (1994). A microcomputercontrolled demand feeder for the study of feeding behavior in fish. Physiology \& Behavior, 55, 1135-1136.

D'amato, M. R., \& Salomon, D. P. (1982). Tune discrimination in monkeys (Cebus apella) and in rats. Animal Learning \& Behavior, 10, 126-134.

FAY, R. R. (1988). Hearing in vertebrates: A psychophysics databook. Winnetka, IL: Hill Fay.

FAY, R. R. (1992). Analytic listening in goldfish. Hearing Research, 59 , 101-107.

Goldman, M., \& Shapiro, S. (1979). Matching-to-sample and oddityfrom-sample in goldfish. Journal of the Experimental Analysis of Behavior, 31, 259-266.

HARRISON, J. M. (1992). Avoiding conflicts between the natural behavior of the animal and the demands of discrimination experiments. Journal of the Acoustical Society of America, 92, 1331-1345.

Horowitz, P., \& HiLl, W. (1990). The art of electronics (2nd ed.). Cambridge: Cambridge University Press.

Magurran, A. (1984, August). Gregarious goldfish. New Scientist, 9 , pp. 32-33.

Neuringer, A., \& Neuringer, M. (1974). Learning by following a food source. Science, 184, 1005-1008.

POPPER, A. N. (1972). Pure-tone auditory thresholds for the carp Cyprinus carpio. Journal of the Acoustical Society of America, 52, 17141717.

Popper, A. N., \& Clarke, N. L. (1976). The auditory system of goldfish (Carassius auratus): Effects of intense acoustic stimulation. Comparative Biochemical Physiology, 53, 11-18. 
Porter, D., \& Neuringer, A. (1984). Music discriminations by pigeons. Journal of Experimental Psychology: Animal Behavior Processes, 10, 138-148.

Segal, M., \& Harrison, J. M. (1978). The control of responding by auditory stimuli: Interactions between different dimensions of stim. uli. Journal of the Experimental Analvsis of Behavior, 30, 97-106.

Stebrins, W. C. (1983). The acoustic sense of animals. Cambridge, MA: Harvard University Press.

WeLtY, J. C. (1934). Experiments in group behavior of fishes. Physiological Zoology, 7, 85-128.

WrIGHT, A. A. (1992). Testing the cognitive capacity of animals. In I. Gormezano \& E. A. Wasserman (Eds.), Learning and memory: The behavioral and biological substrates (pp. 45-60). Hillsdale, NJ: Erlbaum.

WriGht, D. E., \& EAStcotT, A. (1981). Operant conditioning of feeding behavior and patterns of feeding in thick lipped mullet, Cren- imugil labrosus (Risso) and common carp, Cyprinus carpio (L.). Journal of Fish Biology, 20, 625-634.

YAN, H. Y., \& POPPER, A. N. (1991). An automated positive reward method for measuring acoustic sensitivity in fish. Behavior Research Methods, Instruments, \& Computers, 23, 351-356.

YAN, H. Y., \& POPPER, A. N. (1992). Auditory sensitivity of the cichlid fish Astronotus ocellatus (Cuvier). Journal of Comparative Physiology $A, 171,105-109$

ZentalL, T. R., \& Levine, J. M. (1972). Observational learning and social facilitation in the rat. Science, 178, 1220-1221.

(Manuscript received March 30, 1998;

revision accepted for publication November 2, 1998.) 